\title{
NUMERICAL IMPLEMENTATION OF FINITE STRAIN ELASTO-PLASTICITY WITHOUT YIELD SURFACE
}

\author{
CyPRIAN SUCHOCKI \\ Warsaw University of Life Sciences, Department of Fundamental Engineering, Warsaw, Poland \\ e-mail: cyprian_suchocki@sggw.pl
}

\begin{abstract}
In the present study, the finite element (FE) implementation of elasto-plasticity without a yield surface is discussed. For that purpose, the method of perturbing the deformation gradient tensor is employed to calculate approximate tangent moduli. The development of a user subroutine that enables one to use the proposed model within the FE program ABAQUS is covered. A number of exemplary numerical simulations is conducted in order to check the performance of this subroutine. Material parameter values determined for different materials are utilized. Finally, the presented constitutive equation is examined upon its ability to capture the shear-softening process.
\end{abstract}

Keywords: constitutive equation, tangent moduli, UMAT, plasticity

\section{Introduction}

In this study, the finite element (FE) implementation of a plasticity theory is discussed, which does not introduce neither the yield surface nor the split of strain measures into elastic and plastic components. The basic concepts of the considered framework were first introduced as a part of a viscoplastic constitutive model for polymeric materials, cf Suchocki (2015). Further research proved applicability of the proposed theory to model the material response of metals (Suchocki and Skoczylas, 2016). However, in the case of metallic materials, correct numerical implementation of the considered class of constitutive equations requires a completely different approach from the one used in polymer modeling. This new treatment employs the perturbed deformation gradient method and is discussed in detail further in this work.

The proposed framework of constitutive modeling is a special case of the theory of plasticity whose basic assumptions were introduced by Pipkin and Rivlin (1965). However, the concept of an intrinsic time adopted therein and defined as the arclength in the nine-dimensional strain space has been slightly modified for the purpose of present considerations (cf Suchocki, 2015; Suchocki and Skoczylas, 2016). The fictitious time is utilized to build a functional of the deformation history which determines the material stress response and is analogous to the history functional known from the viscoelasticity theory (e.g. Holzapfel, 2010). The whole constitutive model is written in terms of tensorial internal state variables whose change with time is determined by proper evolution equations.

The FE implementation of the flow theory of plasticity based on the Huber-Mises-Hencky $(\mathrm{HMH})$ yield condition (e.g. Olesiak, 1975) has been a subject of research for a long time. Various concepts of numerical integration have been proposed for the HMH flow plasticity such as radial return algorithms, see for instance Simo and Hughes (2000). These algorithms are rather costly in terms of numerical computations. In each iteration of the Newton-Raphson (N-R) procedure, the criterion of material plastic flow has to be checked. If a plastic deformation occurs, another $\mathrm{N}-\mathrm{R}$ iterative process has to be initiated in order to calculate the increment of the equivalent 
plastic strain. Nevertheless, the numerical algorithms and codes for the HMH flow plasticity have been optimized for decades and are considerably well established nowadays.

The FE implementation of plasticity theories which do not introduce a yield surface attracted rather little attention over the years. Lee (1995) discussed numerical implementation of the endochronic theory of plasticity that was developed by Valanis (1971a,b). Kästner et al. (2012) developed and implemented into the finite element method (FEM) a viscoplastic constitutive model which contained an elasto-plastic component similar to the endochronic formulation by Valanis. Both aforementioned approaches referred to the small strain domain. The constitutive equation proposed by Kästner et al. (2012) was further extended to the finite strain range by Alkas Yonan et al. (2013) using the spatial description in the deformed configuration of material continuum. The FE implementation was discussed as well. A viscoplastic model containing an elasto-plastic term without a yield condition was also proposed and implemented into FEM by Suchocki (2015). This model was formulated in the reference configuration using the material description and presents itself a generalization of the hyperelasticity theory. The approximation of the Material Jacobian (MJ) tensor proposed by Suchocki (2015) proved to be accurate enough for the purpose of modeling the mechanical response of polymers. However, application of the same constitutive modeling framework to metallic materials results in much higher values of the model constants. Consequently, it was noticed that a more accurate approximation of the MJ is required in order to describe the mechanical behavior of metals.

In the present work, the perturbed deformation gradient method proposed by Sun et al. (2008) is utilized to implement into FEM the constitutive equation of elasto-plasticity without the yield surface, cf Suchocki an Skoczylas (2016). The implementation of the model into the FE program ABAQUS is facilitated by taking advantage of the user subroutine UMAT (UserMATerial). The presented framework can also be adapted to other FE packages. The accuracy of the implementation is checked using numerous FE simulations performed for different material parameter values. The simplified approach which can be used for the modeling of polymeric materials such as polyethylene, for instance, is briefly discussed as well. The ability of the proposed constitutive model to describe the shear-softening process (e.g. Kowalewski and Szymczak, 2009) is investigated. Finally, an exemplary simulation is conducted to check the performance of the user subroutine for the case of nonhomogenous stress and strain states.

\section{Basic notions}

The stored elastic energy function $W(\mathbf{C})$ is assumed in the decoupled form (e.g. Holzapfel, 2010)

$$
W(\mathbf{C})=U(J)+\bar{W}(\overline{\mathbf{C}})
$$

where $U(J)$ and $\bar{W}(\overline{\mathbf{C}})$ are the volumetric and isochoric components, respectively. The multiplicative split of the deformation gradient $\mathbf{F}$ is utilized, i.e.

$$
\mathbf{F}=\mathbf{F}_{\text {vol }} \overline{\mathbf{F}} \quad J=\operatorname{det} \mathbf{F} \quad \mathbf{F}_{\text {vol }}=J^{\frac{1}{3}} \mathbf{1} \quad \overline{\mathbf{F}}=J^{-\frac{1}{3}} \mathbf{F}
$$

which enables one to define the isochoric right Cauchy-Green (C-G) deformation tensor $\overline{\mathbf{C}}$ with a set of algebraic invariants, i.e.

$$
\overline{\mathbf{C}}=\overline{\mathbf{F}}^{\mathrm{T}} \overline{\mathbf{F}}=J^{-\frac{2}{3}} \mathbf{C} \quad \bar{I}_{1}=\operatorname{tr} \overline{\mathbf{C}} \quad \bar{I}_{2}=\frac{1}{2}\left[(\operatorname{tr} \overline{\mathbf{C}})^{2}-\operatorname{tr} \overline{\mathbf{C}}^{2}\right] \quad \bar{I}_{3}=\operatorname{det} \overline{\mathbf{C}}=1
$$

The total second Piola-Kirchhoff (P-K) stress $\mathbf{S}$ is assumed to be the sum of the elastic component $\mathbf{S}_{0}$ and the tensorial internal state variables $\widetilde{\mathbf{H}}_{k}(k=1,2, \ldots, N)$ which account for the inelastic effects (cf Suchocki and Skoczylas, 2016), thus 


$$
\begin{array}{lr}
\mathbf{S}=\mathbf{S}_{0}+\sum_{k=1}^{N} \widetilde{\mathbf{H}}_{k} & \mathbf{S}_{0}=\mathbf{S}_{0}^{v o l}+\mathbf{S}_{0}^{i s o} \\
\mathbf{S}_{0}^{v o l}=J p \mathbf{C}^{-1} & \mathbf{S}_{0}^{i s o}=J^{-\frac{2}{3}} \operatorname{DEV}[\overline{\mathbf{S}}]
\end{array}
$$

where

$$
p=\frac{\partial U}{\partial J} \quad \overline{\mathbf{S}}=\left.2 \frac{\partial \bar{W}}{\partial \overline{\mathbf{C}}}\right|_{\overline{\mathbf{C}}=\overline{\mathbf{C}}^{\mathrm{T}}} \quad \operatorname{DEV}[\overline{\mathbf{S}}]=\overline{\mathbf{S}}-\frac{1}{3}(\overline{\mathbf{S}} \cdot \overline{\mathbf{C}}) \overline{\mathbf{C}}^{-1}
$$

The change of each of the internal state variables is governed by a separate evolution equation, i.e.

$$
\dot{\widetilde{\mathbf{H}}}_{k}+\frac{1}{\widetilde{D}_{k} M(|\dot{\overline{\mathbf{C}}}|)} \widetilde{\mathbf{H}}_{k}=\gamma_{k} \dot{\mathbf{S}}_{0}^{i s o} \quad k=1,2, \ldots, N
$$

with

$$
M(|\dot{\mathbf{C}}|)=\bar{J}_{2}^{-\frac{1}{2}} \quad \bar{J}_{2}=\operatorname{tr} \dot{\overline{\mathbf{C}}}^{2}
$$

whereas $\widetilde{D}_{k}$ and $\gamma_{k}$ are the material parameters $k=1,2, \ldots, N$.

\section{Finite element implementation}

For the purpose of numerical implementation, the considered constitutive model has to be discretized and further linearized, i.e. expressed as a linear relation between the finite increments of stress and strain. Below both formulations are presented.

\subsection{Discretized constitutive equation}

According to Eqs. $(2.4)_{1}$ and $(2.4)_{2}$, for the time increment $n+1$, the total second P-K stress is given as

$$
\mathbf{S}_{n+1}=\mathbf{S}_{0 n+1}+\sum_{k=1}^{N} \widetilde{\mathbf{H}}_{k n+1} \quad \mathbf{S}_{0 n+1}=\mathbf{S}_{0 n+1}^{v o l}+\mathbf{S}_{0 n+1}^{i s o}
$$

with the internal state variables being determined from the discretized evolution equations, i.e.

$$
\widetilde{\mathbf{H}}_{k n+1}=\frac{\left(1-\frac{1}{\widetilde{D}_{k}} \frac{\Delta z_{n+1}}{2}\right) \widetilde{\mathbf{H}}_{k n}+\gamma_{k}\left(\mathbf{S}_{0 n+1}^{i s o}-\mathbf{S}_{0 n}^{i s o}\right)}{1+\frac{1}{\widetilde{D}_{k}} \frac{\Delta z_{n+1}}{2}} \quad k=1,2, \ldots, N
$$

where $\Delta z_{n+1}$ is the increment of arclength, namely

$$
\Delta z_{n+1}=\sqrt{\Delta \overline{\mathbf{C}}_{n+1} \cdot \Delta \overline{\mathbf{C}}_{n+1}}
$$

The recurrence-update formula defined by Eq. (3.2) has been obtained by applying the central difference rule to Eq. (2.6), cf Suchocki (2015). 


\subsection{Linearized constitutive model}

Taking the directional derivative on Eq. (3.1) 1 yields

$$
\Delta \mathbf{S}_{n+1}=\mathcal{C}_{n+1}^{e-p} \cdot \frac{1}{2} \Delta \mathbf{C}_{n+1} \quad \mathcal{C}_{n+1}^{e-p}=\left.2 \frac{\partial \mathbf{S}_{n+1}}{\partial \mathbf{C}_{n+1}}\right|_{\mathbf{C}_{n+1}=\mathbf{C}_{n+1}^{\mathrm{T}}}
$$

with $\mathcal{C}_{n+1}^{e-p}$ being the fourth-order elasto-plastic stiffness tensor. Pushing Eq. (3.4) ${ }_{1}$ forward to the current configuration leads to the incremental rate equation

$$
\tau_{n+1}^{\nabla}=J_{n+1} \mathcal{C}_{n+1}^{M J} \cdot \Delta \mathbf{D}_{n+1}
$$

where the incremental Zaremba-Jaumann (Z-J) rate of the Kirchhoff stress $\boldsymbol{\tau}_{n+1}$ is given as

$$
\boldsymbol{\tau}_{n+1}^{\nabla}=\Delta \boldsymbol{\tau}_{n+1}-\Delta \mathbf{W}_{n+1} \boldsymbol{\tau}_{n+1}-\boldsymbol{\tau}_{n+1} \Delta \mathbf{W}_{n+1}^{\mathrm{T}} \quad \boldsymbol{\tau}_{n+1}=\mathbf{F}_{n+1} \mathbf{S}_{n+1} \mathbf{F}_{n+1}^{\mathrm{T}}
$$

The increments of the spin tensor $\mathbf{W}_{n+1}$, the strain rate tensor $\mathbf{D}_{n+1}$ and the deformation gradient $\mathbf{F}_{n+1}$ are defined by the following formulas

$$
\begin{aligned}
& \Delta \mathbf{W}_{n+1}=\frac{1}{2}\left[\Delta \mathbf{F}_{n+1} \mathbf{F}_{n+1}^{-1}-\left(\Delta \mathbf{F}_{n+1} \mathbf{F}_{n+1}^{-1}\right)^{\mathrm{T}}\right] \\
& \Delta \mathbf{D}_{n+1}=\frac{1}{2}\left[\Delta \mathbf{F}_{n+1} \mathbf{F}_{n+1}^{-1}+\left(\Delta \mathbf{F}_{n+1} \mathbf{F}_{n+1}^{-1}\right)^{\mathrm{T}}\right] \\
& \Delta \mathbf{F}_{n+1}=\mathbf{F}_{n+1} \mathbf{F}_{n}^{-1}
\end{aligned}
$$

whereas the MJ tensor takes the form

$$
\mathcal{C}_{n+1}^{M J}=\frac{1}{J_{n+1}}\left(\mathcal{C}_{n+1}^{\tau c}+\mathcal{I}_{n+1}\right)
$$

with

$$
\begin{aligned}
& \mathcal{C}_{n+1}^{\tau c}=\left(F_{i P} F_{j Q} F_{k R} F_{l S} \mathcal{C}_{P Q R S}^{e-p}\right)_{n+1} \mathbf{e}_{i} \otimes \mathbf{e}_{j} \otimes \mathbf{e}_{k} \otimes \mathbf{e}_{l} \\
& \mathcal{I}_{n+1}=\frac{1}{2}\left(\delta_{i k} \tau_{j l}+\tau_{i k} \delta_{j l}+\delta_{i l} \tau_{j k}+\tau_{i l} \delta_{j k}\right)_{n+1} \mathbf{e}_{i} \otimes \mathbf{e}_{j} \otimes \mathbf{e}_{k} \otimes \mathbf{e}_{l}
\end{aligned}
$$

where $\left\{\mathbf{e}_{k}\right\}(k=1,2,3)$ is a Cartesian base in the current configuration. For the class of constitutive equations considered in this work, it is not possible to find an analytical, closed-form equation determining the MJ tensor. Thus, approximation methods have to be employed.

\subsection{Approximate formula for Material Jacobian}

Inserting Eq. (3.1) 1 into Eq. (3.4) 2 yields

$$
\mathcal{C}_{n+1}^{e-p}=\left.2 \frac{\partial \mathbf{S}_{n+1}}{\partial \mathbf{C}_{n+1}}\right|_{\mathbf{C}_{n+1}=\mathbf{C}_{n+1}^{\mathrm{T}}}=\left.2 \frac{\partial \mathbf{S}_{0 n+1}}{\partial \mathbf{C}_{n+1}}\right|_{\mathbf{C}_{n+1}=\mathbf{C}_{n+1}^{\mathrm{T}}}+\left.\sum_{k=1}^{M} 2 \frac{\partial \widetilde{\mathbf{H}}_{k n+1}}{\partial \mathbf{C}_{n+1}}\right|_{\mathbf{C}_{n+1}=\mathbf{C}_{n+1}^{\mathrm{T}}}
$$

where, according to $(3.1)_{2}$, the elasticity tensor $\mathcal{C}_{0} n+1$ is given as

$$
\begin{aligned}
& \mathcal{C}_{0 n+1}=\left.2 \frac{\partial \mathbf{S}_{0 n+1}}{\partial \mathbf{C}_{n+1}}\right|_{\mathbf{C}_{n+1}=\mathbf{C}_{n+1}^{\mathrm{T}}}=\left.2 \frac{\partial \mathbf{S}_{0 n+1}^{v o l}}{\partial \mathbf{C}_{n+1}}\right|_{\mathbf{C}_{n+1}=\mathbf{C}_{n+1}^{\mathrm{T}}}+\left.2 \frac{\partial \mathbf{S}_{0 n+1}^{i s o}}{\partial \mathbf{C}_{n+1}}\right|_{\mathbf{C}_{n+1}=\mathbf{C}_{n+1}^{\mathrm{T}}} \\
& =\mathcal{C}_{0 n+1}^{v o l}+\boldsymbol{C}_{0 n+1}^{i s o}
\end{aligned}
$$

In general, utilizing Eq. (3.2) for calculation of $\left.2 \frac{\partial \widetilde{\mathbf{H}}_{k n+1}}{\partial \mathbf{C}_{n+1}}\right|_{\mathbf{C}_{n+1}=\mathbf{C}_{n+1}^{\mathrm{T}}}$ derivatives $(k=1,2, \ldots, N)$ leads the approximate elasto-plastic stiffness tensor $\mathcal{C}_{n+1}^{e-p}$ to the loss of certain symmetries which 
are required for a material to be initially isotropic. This fact was previously highlighted by Kästner et al. (2012) for the case of infinitesimal strains. However, for small values of constants $\gamma_{k}$ $(k=1,2, \ldots, N)$, the following approximation provides a good accuracy and preserves the initial material isotropy, i.e.

$$
\left.2 \frac{\partial \widetilde{\mathbf{H}}_{k n+1}}{\partial \mathbf{C}_{n+1}}\right|_{\mathbf{C}_{n+1}=\mathbf{C}_{n+1}^{\mathrm{T}}} \approx \gamma_{k}\left(1+\frac{\Delta z_{n+1}}{2 \widetilde{D}_{k}}\right)^{-1} \mathcal{C}_{0 n+1}^{i s o}
$$

Substitution of Eqs (3.11) and (3.12) into Eq. (3.10) leads to the formula

$$
\mathcal{C}_{n+1}^{e-p}=\mathcal{C}_{0 n+1}^{\text {vol }}+\left[1+\sum_{k=1}^{N} \gamma_{k}\left(1+\frac{\Delta z_{n+1}}{2 \widetilde{D}_{k}}\right)^{-1}\right] \mathcal{C}_{0 n+1}^{i s o}
$$

An analogous result can be found for the small strain formulation, cf Kästner et al. (2012). By pushing Eq. (3.13) forward to the current configuration, the stiffness tensor associated with the convected rate of the Kirchhoff stress is found, i.e.

$$
\mathcal{C}_{n+1}^{\tau c}=\mathcal{C}_{n+1}^{\text {vol }}+\left[1+\sum_{k=1}^{N} \gamma_{k}\left(1+\frac{\Delta z_{n+1}}{2 \widetilde{D}_{k}}\right)^{-1}\right] \mathcal{C}_{n+1}^{i s o}
$$

By inserting Eq. (3.14) into Eq. (3.8), the required form of the MJ is obtained. The discretized form of the constitutive equation along with the approximate MJ have been implemented into the FE program ABAQUS by utilizing the user subroutine UMAT written in FORTRAN 77 (cf Hibbit et al., 2008).

\subsection{Application to polymeric materials}

Below the accuracy of MJ approximation defined by Eq. (3.14) is tested for the material constants that have been determined for ultra-high molecular weigth polyethylene (UHMWPE) using the previously developed parameter evaluation algorithm (Suchocki and Skoczylas, 2016), cf Table 1. The isochoric stored-energy function $\bar{W}$ is chosen in the form proposed by Knowles (1977), whereas the volumetic function $U$ is assumed in the form used by Sussman and Bathe (1987), i.e.

$$
\bar{W}=\frac{\mu}{2 b}\left\{\left[1+\frac{b}{\kappa}\left(\bar{I}_{1}-3\right)\right]^{\kappa}-1\right\} \quad U=\frac{1}{D_{1}}(J-1)^{2}
$$

Furthermore, it is assumed that the inelastic effects are modeled by a single internal variable $(N=1)$.

Table 1. Material constitutive parameters

\begin{tabular}{|l|c|c|c|c|c|c|}
\hline \multicolumn{1}{|c|}{ Material } & $\mu[\mathrm{MPa}]$ & $b[-]$ & $\kappa[-]$ & $D_{1}\left[\mathrm{MPa}^{-1}\right]$ & $\gamma_{1}[-]$ & $\widetilde{D}_{1}[-]$ \\
\hline \hline UHMWPE & 62.042 & 3.63 & 0.25 & $3.3 \mathrm{E}-8$ & 2.83 & $29 \mathrm{E}-3$ \\
\hline Cast iron & 665.16 & - & - & $3.3 \mathrm{E}-8$ & 57.40 & $22 \mathrm{E}-3$ \\
\hline Brass & 703.48 & - & - & $1 \mathrm{E}-5$ & 48.92 & $92 \mathrm{E}-4$ \\
\hline WCL & 1249.54 & - & - & $1 \mathrm{E}-5$ & 31.73 & $34 \mathrm{E}-3$ \\
\hline Inconel & 1114.68 & - & - & $3.3 \mathrm{E}-8$ & 83.43 & $34 \mathrm{E}-4$ \\
\hline
\end{tabular}

The test simulation involves a polymeric $1 \mathrm{~mm} \times 1 \mathrm{~mm} \times 1 \mathrm{~mm}$ block undergoing ramp loading and unloading (cf Fig. 1). The excitation is kinematic in the form of a prescribed displacement $\Delta \mathbf{u}_{1}$ of the cube frontal face along direction "1". This results in the stretch ratios $\lambda_{1}$ and $\lambda_{2}$ in direction "1" and perpendicular directions " 2 " and "3", respectively. The boundary conditions 
along with the undeformed and deformed configurations are illustrated in Fig. 1. The polymeric block is first meshed using a single $\mathrm{C} 3 \mathrm{D} 8 \mathrm{H}^{1}$ element. The simulations are then repeated with the mesh of 125 elements with the same results. The tests have been performed for both positive and negative magnitude of $\Delta \mathbf{u}_{1}$ (elongation and shortening, respectively).
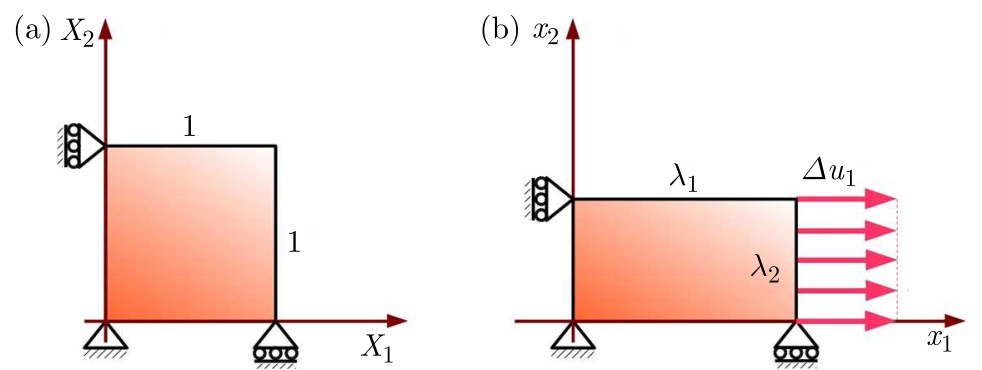

Fig. 1. Uniaxial tension-compression simulation: (a) undeformed FE, (b) deformed FE

In order to verify the results obtained in FE simulations, a Scilab program has been utilized which enables one to solve the one-dimensional process equations. This program is based on the previously presented scalar equation system, cf Suchocki and Skoczylas (2016). As it can be seen in Fig. 2, the ABAQUS UMAT and Scilab results are in an excellent agreement for both tension and compression which allows one to conclude that for small values of $\gamma_{k}$ constants $(k=1,2, \ldots, N)$ Eq. (3.14) provides an accurate approximation of the elasto-plastic stiffness.

(a)

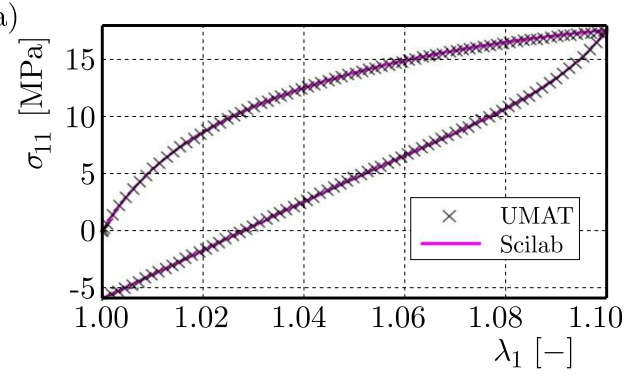

(b)

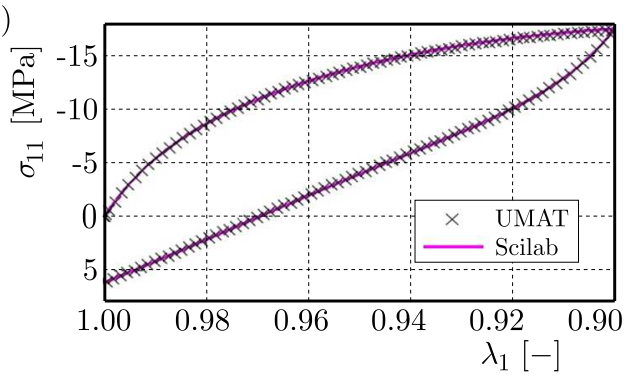

Fig. 2. Polymeric block undergoing: (a) ramp tensions with unloading, (b) ramp compression with unloading

\subsection{Perturbed deformation gradient approach}

For the group of metallic materials, the model parameters reach considerably high values, which makes approximate Eq. (3.14) inaccurate. Thus, the method of computing the approximate tangent moduli is utilized which is based on a perturbation of the deformation gradient tensor. This technique was originally proposed by Miehe (1996) and was further extended by Sun et al. (2008).

The perturbed deformation gradient tensor in the time increment $n+1$ is defined as

$$
\widehat{\mathbf{F}}_{n+1}^{(k l)}=\mathbf{F}_{n+1}+\Delta \mathbf{F}_{n+1}^{(k l)}
$$

where

$$
\Delta \mathbf{F}_{n+1}^{(k l)}=\frac{\varepsilon}{2}\left(\mathbf{e}_{k} \otimes \mathbf{e}_{l} \mathbf{F}_{n+1}+\mathbf{e}_{l} \otimes \mathbf{e}_{k} \mathbf{F}_{n+1}\right)
$$

with $\varepsilon$ being the perturbation parameter. The increments of the strain rate tensor $\mathbf{D}_{n+1}$ and the spin tensor $\mathbf{W}_{n+1}$ due to the deformation gradient increment $\Delta \mathbf{F}_{n+1}^{(k l)}$ are given by the following relationships

\footnotetext{
${ }^{1}$ Cubic, three-dimensional, 8 nodes, hybrid.
} 


$$
\begin{aligned}
& \Delta \mathbf{D}_{n+1}^{(k l)}=\frac{1}{2}\left[\Delta \mathbf{F}_{n+1}^{(k l)} \mathbf{F}_{n+1}^{-1}+\left(\Delta \mathbf{F}_{n+1}^{(k l)} \mathbf{F}_{n+1}^{-1}\right)^{\mathrm{T}}\right] \\
& \Delta \mathbf{W}_{n+1}^{(k l)}=\frac{1}{2}\left[\Delta \mathbf{F}_{n+1}^{(k l)} \mathbf{F}_{n+1}^{-1}-\left(\Delta \mathbf{F}_{n+1}^{(k l)} \mathbf{F}_{n+1}^{-1}\right)^{\mathrm{T}}\right]
\end{aligned}
$$

Inserting Eq. (3.17) into Eqs (3.18) and performing the computations results in

$$
\Delta \mathbf{D}_{n+1}^{(k l)}=\frac{\varepsilon}{2}\left(\mathbf{e}_{k} \otimes \mathbf{e}_{l}+\mathbf{e}_{l} \otimes \mathbf{e}_{k}\right) \quad \Delta \mathbf{W}_{n+1}^{(k l)}=\mathbf{0}
$$

The increment of the Kirchhoff stress $\boldsymbol{\tau}_{n+1}$ is approximated by a finite difference, i.e.

$$
\Delta \boldsymbol{\tau}_{n+1} \approx \boldsymbol{\tau}_{n+1}\left(\widehat{\mathbf{F}}_{n+1}^{(k l)}\right)-\boldsymbol{\tau}_{n+1}\left(\mathbf{F}_{n+1}\right)
$$

Substitution of Eqs (3.19) and Eq. (3.20) into Eqs (3.5) and (3.6) 1 yields

$$
\boldsymbol{\tau}_{n+1}\left(\widehat{\mathbf{F}}_{n+1}^{(k l)}\right)-\boldsymbol{\tau}_{n+1}\left(\mathbf{F}_{n+1}\right) \approx J_{n+1} \mathcal{C}_{n+1}^{M J} \cdot \frac{\varepsilon}{2}\left(\mathbf{e}_{k} \otimes \mathbf{e}_{l}+\mathbf{e}_{l} \otimes \mathbf{e}_{k}\right)
$$

which, in turn, leads to the approximate formula for the components of MJ

$$
\mathcal{C}_{i j k l}^{M J}=\frac{1}{J_{n+1} \varepsilon}\left[\boldsymbol{\tau}_{n+1}\left(\widehat{\mathbf{F}}_{n+1}^{(k l)}\right)-\boldsymbol{\tau}_{n+1}\left(\mathbf{F}_{n+1}\right)\right]_{i j}
$$

In order to compute all 21 independent components of $\mathcal{C}_{n+1}^{M J}$, the deformation gradient tensor has to be perturbed for 6 times. During the entire procedure, the parameter $\varepsilon$ is held constant with its value being prescribed by the programmer.

The framework presented above has been utilized to implement the proposed constitutive equation of elasto-plasticity into the FE package ABAQUS. A proper user subroutine UMAT written in FORTRAN 77 was used for that purpose, cf Hibbit et al. (2008). In the case of all the calculations described in this work $\varepsilon=1 \mathrm{E}-8$ was assumed as recommended by Sun et al. (2008).

\subsection{User subroutine UMAT}

The perturbed deformation gradient method requires calculation of the Kirchhoff stress tensor components for a number of different deformation gradients. Thus, in order to shorten the UMAT code, a separate subroutine CALCSTRESS computing the stress tensor has been written and is called within the main code whenever it is necessary to compute the stress. The user subroutine is then used to simulate the mechanical response of metals. The structure of the code is depicted in the attached scheme.

\section{Application to metallic materials}

For the purpose of modeling the constitutive response of metals, it has been assumed that the isochoric stored energy had the form of neo-Hooke function, i.e.

$$
\bar{W}=\frac{\mu}{2}\left(\bar{I}_{1}-3\right)
$$

with the volumetric energy component given by Eq. $(3.15)_{2}$ which reflects a linear relationship between the pressure and the relative volume change. A more detailed description of the volumetric material response can be achieved by the adjustment of a more sophisticated volumetric energy $U$ (cf Doll and Schweizerhof, 2000). However, such an approach would require a more thorough experimental study.

The material parameter evaluation algorithm developed by Suchocki and Skoczylas (2016) has been utilized to determine the constant values for: cast iron, brass, WCL steel and Inconel. 
Algorithm for the implementation in Abaqus

Subroutine UMAT(input: $\mathbf{F}_{n+1}$, STATEV, PROPS; output: $\boldsymbol{\sigma}_{n+1}, \mathcal{C}_{n+1}^{M J}$ )

1. Calculate transformation Jacobian $J_{n+1}=\operatorname{det} \mathbf{F}_{n+1}$

2. Extract STATEV, i.e. $\mathbf{S}_{0 n}^{i s o}, \widetilde{\mathbf{H}}_{k n}, \overline{\mathbf{C}}_{n}(k=1,2, \ldots, N)$

3. Calculate Cauchy stress: Call CALCSTRESS, $\boldsymbol{\sigma}_{n+1}=J_{n+1}^{-1} \boldsymbol{\tau}_{n+1}$

Subroutine CALCSTRESS(input: $\mathbf{F}_{n+1}, J_{n+1}$, STATEV, PROPS; output: $\left.\boldsymbol{\tau}_{n+1}\right)$

(a) Calculate strain measures from current increment

$$
\mathbf{C}_{n+1}=\mathbf{F}_{n+1}^{\mathrm{T}} \mathbf{F}_{n+1} \quad \overline{\mathbf{C}}_{n+1}=J_{n+1}^{-\frac{2}{3}} \mathbf{C}_{n+1}
$$

(b) Calculate elastic stresses from current increment

$$
\begin{array}{ll}
\mathbf{S}_{0 n+1}^{v o l}=J_{n+1} p_{n+1} \mathbf{C}_{n+1}^{-1} & p_{n+1}=\partial_{J_{n+1}} U\left(J_{n+1}\right) \\
\mathbf{S}_{0 n+1}^{i s o}=J_{n+1}^{-\frac{2}{3}} \operatorname{DEV}\left[\overline{\mathbf{S}}_{n+1}\right] & \overline{\mathbf{S}}_{n+1}=\left.2 \partial_{\overline{\mathbf{C}}_{n+1}} \bar{W}\left(\overline{\mathbf{C}}_{n+1}\right)\right|_{\overline{\mathbf{C}}_{n+1}=\overline{\mathbf{C}}_{n+1}^{\mathrm{T}}} \\
\mathbf{S}_{0 n+1}=\mathbf{S}_{0 n+1}^{v o l}+\mathbf{S}_{0 n+1}^{i s o} &
\end{array}
$$

(c) Update internal state variables $(k=1,2, \ldots, N)$

$$
\begin{aligned}
\Delta z_{n+1} & =\sqrt{\Delta \overline{\mathbf{C}}_{n+1} \cdot \Delta \overline{\mathbf{C}}_{n+1}} \quad \Delta \overline{\mathbf{C}}_{n+1}=\overline{\mathbf{C}}_{n+1}-\overline{\mathbf{C}}_{n} \\
\widetilde{\mathbf{H}}_{k n+1} & =\frac{\left(1-\frac{1}{\widetilde{D}_{k}} \frac{\Delta z_{n+1}}{2}\right) \widetilde{\mathbf{H}}_{k n}+\gamma_{k}\left(\mathbf{S}_{0 n+1}^{i s o}-\mathbf{S}_{0 n}^{i s o}\right)}{1+\frac{1}{\widetilde{D}_{k}} \frac{\Delta z_{n+1}}{2}}
\end{aligned}
$$

(d) Calculate total stress from current increment

$$
\mathbf{S}_{n+1}=\mathbf{S}_{0 n+1}+\sum_{k=1}^{M} \widetilde{\mathbf{H}}_{k n+1} \quad \boldsymbol{\tau}_{n+1}=\mathbf{F}_{n+1} \mathbf{S}_{n+1} \mathbf{F}_{n+1}^{\mathrm{T}}
$$

4. Calculate elastic-plastic stiffness: Call CALCTANGENT

Subroutine CALCTANGENT(input: $\mathbf{F}_{n+1}, J_{n+1}$, STATEV, PROPS, $\boldsymbol{\tau}_{n+1}$; output: $\left.\mathcal{C}_{n+1}^{M J}\right)$

For prescribed $i j$ and $r s$ :

(a) Call DEFGRAD

Subroutine DEFGRAD(input: $\mathbf{F}_{n+1}, r s$; output: $\widehat{\mathbf{F}}_{n+1}^{(r s)}$ )

i. Calculate deformation gradient increment

$$
\Delta \mathbf{F}_{n+1}^{(r s)}=\frac{\varepsilon}{2}\left(F_{s L} \mathbf{e}_{r} \otimes \mathbf{e}_{L}+F_{r L} \mathbf{e}_{s} \otimes \mathbf{e}_{L}\right)
$$

ii. Calculate perturbed deformation gradient

$$
\widehat{\mathbf{F}}_{n+1}^{(r s)}=\mathbf{F}_{n+1}+\Delta \mathbf{F}_{n+1}^{(r s)}
$$

(b) Call CALCSTRESS ( $\widehat{\mathbf{F}}_{n+1}^{(r s)}$ as input deformation gradient)

(c) Calculate Material Jacobian components:

$$
\mathcal{C}_{i j r s}^{M J}=\frac{1}{J_{n+1} \varepsilon}\left[\boldsymbol{\tau}_{n+1}\left(\widehat{\mathbf{F}}_{n+1}^{(r s)}\right)-\boldsymbol{\tau}_{n+1}\left(\mathbf{F}_{n+1}\right)\right]_{i j}
$$

5. Store $\mathbf{S}_{0 n+1}^{i s o}, \widetilde{\mathbf{H}}_{k n+1}, \overline{\mathbf{C}}_{n+1}$ in $\operatorname{STATEV}(k=1,2, \ldots, N)$ 
(a)

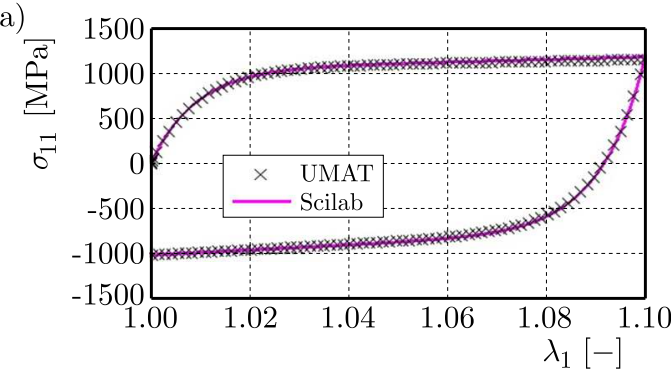

(c)

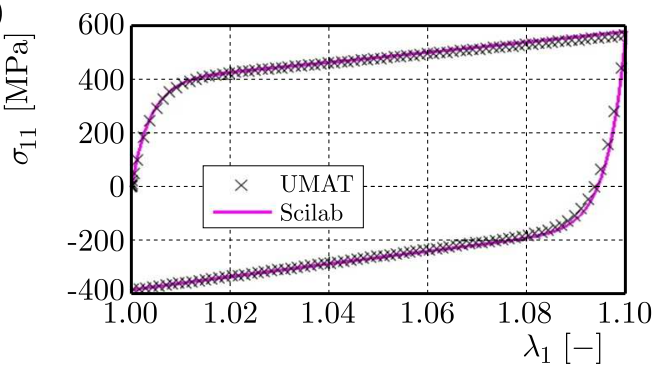

(e)

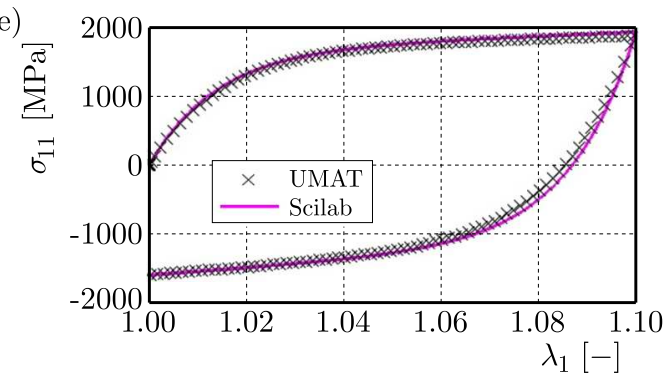

(g)

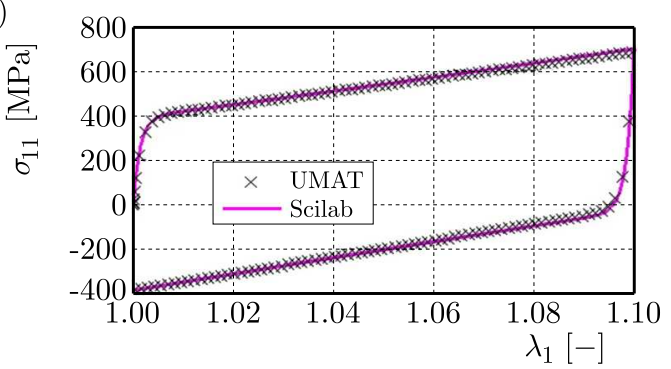

(b)

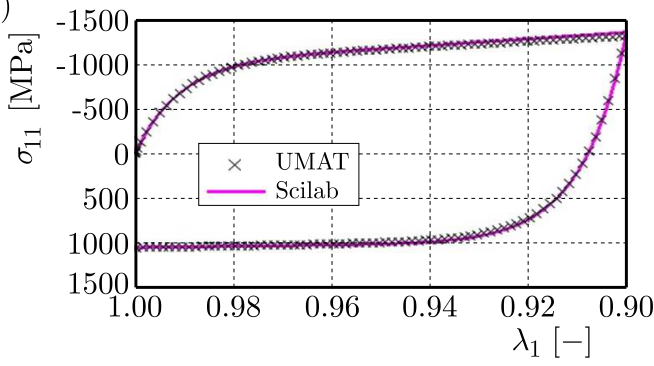

(d)

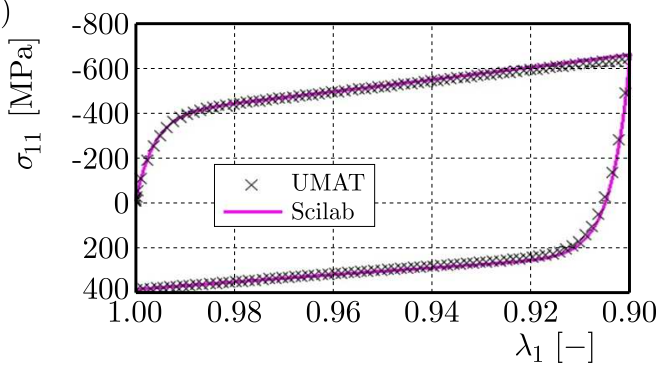

(f)

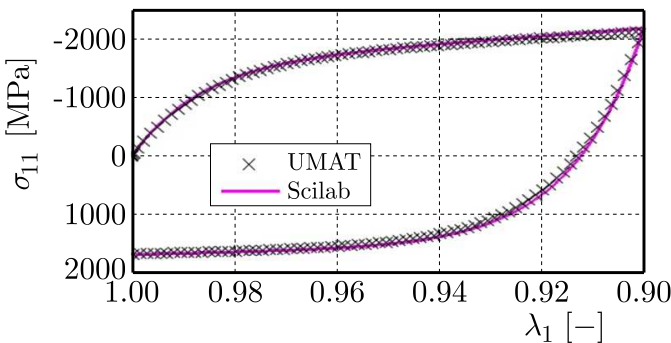

(h)

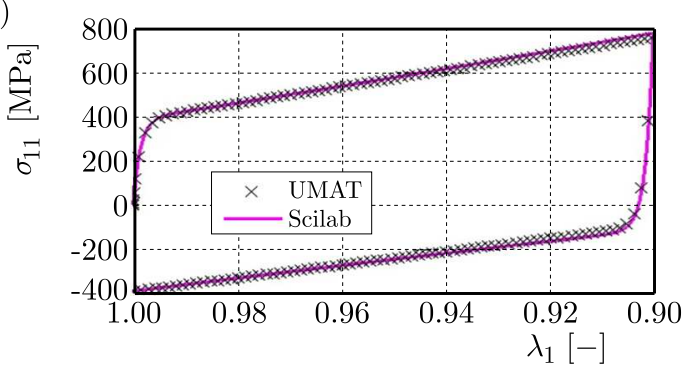

Fig. 3. Metal block undergoing ramp loading-unloading: (a) cast iron tension, (b) cast iron compression, (c) brass tension, (d) brass compression, (e) WCL tension, (f) WCL compression, (g) Inconel tension, (h) Inconel compression

The parameter values have been gathered in Table 1. In simple tension experiments, metals usually act like incompressible materials after reaching the axial strain value of $0.5 \%$ with their Poisson's ratio close to 0.5 (e.g. Kozłowska, 2011). Thus, the incompressibility condition is well established within the theory of plasticity. In order to account for the material near incompressibility of cast iron and Inconel, the hybrid elements $\mathrm{C} 3 \mathrm{D} 8 \mathrm{H}$ have been used during numerical simulations. In order to check the performance of the UMAT code in the presence of a certain amount of compressibility, $D_{1}=\sim 1 \mathrm{E}-5 \mathrm{MPa}^{-1}$ has been set for brass and WCL steel. In the case of the last two materials, ordinary cubic elements C3D8 were utilized.

For each of the considered materials ramp loading/unloading processes have been simulated. Both tension and compression tests have been considered. The boundary conditions are depicted in Fig. 1. In parallel to the FE simulations, every considered process has been independently simulated in Scilab by direct integration of the one-dimensional process equations (cf 
Suchocki and Skoczylas, 2016). An excellent agreement has been found between ABAQUS and Scilab predictions which is depicted in Fig. 3 and allows one to conclude that the utilized MJ approximation method provides satisfying accuracy.

\section{Comparison to HMH plasticity}

In order to highlight the benefits of the proposed formulation of elasto-plasticity, a comparative study has been conducted. The material parameters of the HMH plasticity with isotropic hardening were determined for brass using the same experimental data that were utilized to evaluate the constants of the proposed new constitutive equation.

The determined parameters of the HMH plasticity were defined in ABAQUS to perform a simulation of ramp tension with unloading. The large strain formulation was utilized by setting on the NLGEOM option. Again, the boundary conditions illustrated in Fig. 1 were used. The results along with the list of material constants can be seen in Fig. 4a. The flow plasticity model provides an accurate description of the monotonic loading process, however, at the price of defining 16 material parameters (Fig. 4b). No Bauschinger effect has been observed.

(a)

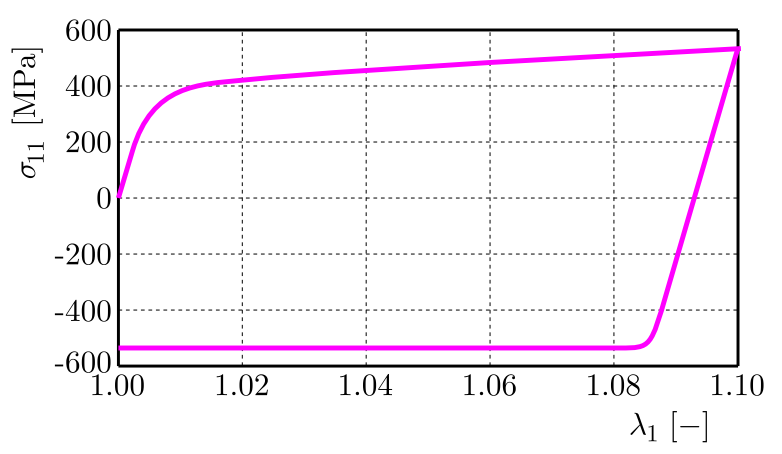

(b)

\begin{tabular}{|c|c|c|}
\hline \multicolumn{2}{|c|}{$E[\mathrm{MPa}]$} & $\nu[-]$ \\
\hline \multicolumn{2}{|c|}{84298} & 0.36 \\
\hline$i$ & $\sigma_{y}\left(e_{i}^{p}\right)$ & $e_{i}^{p}$ \\
\hline 1 & 218 & 0 \\
\hline 2 & 311 & 0.00141 \\
\hline 3 & 374 & 0.004 \\
\hline 4 & 402 & 0.00729 \\
\hline 5 & 430 & 0.0193 \\
\hline 6 & 470 & 0.0412 \\
\hline 7 & 538 & 0.0935 \\
\hline
\end{tabular}

Fig. 4. HMH plasticity model for brass: (a) ramp tension-compression response, (b) material parameters

In the next step, the HMH flow plasticity and the proposed elasto-plastic model have been compared in their ability to describe the shear-softening process (e.g. Kowalewski and Szymczak, 2009). For that purpose, FE simulations were prepared in which a brass block with the dimensions $1 \mathrm{~mm} \times 1 \mathrm{~mm} \times 1 \mathrm{~mm}$ was undergoing simultaneous elongation and shear deformation. The initial configuration of the block can be seen in Fig. 5d. The simulation was divided into two stages, i.e. uniaxial tensile prestrain (stage one) and combined tension and shear (stage two). For the first stage of the simulation, the following boundary conditions have been assumed (cf Fig. 5e):

1. The displacement in "1" direction is set to zero on the face $A B C D$.

2. The displacement in "2" direction is set to zero on the face $A A^{\prime} B B^{\prime}$.

3. The displacement in " 3 " direction is set to zero on the face $B B^{\prime} C C^{\prime}$.

4. On the face $A^{\prime} B^{\prime} C^{\prime} D^{\prime}$, a ramp displacement in "1" direction is defined with the maximum value $\Delta u_{1} / 2=0.0275 \mathrm{~mm}$.

The uniaxial deformation phase ends when $t=t_{0}$ (Fig. 5e). For the second stage of the simulation the boundary conditions listed below are assumed:

1. The displacement in "1" direction is set to zero on the edge $D C$.

2. The zero displacement is set for the point $B$. 
3. The displacements in "1" and "2" directions are set to zero for the point $A$.

4. The zero displacement in "3" direction is set for the edge $B^{\prime} C^{\prime}$.

5. The displacements in "1" and "2" directions are imposed on the face $A^{\prime} B^{\prime} C^{\prime} D^{6}$. The displacement in "1" direction is assumed to increase linearly strating from the initial value $\Delta u_{1} / 2=0.0275 \mathrm{~mm}$ up to the maximum value $\Delta u_{1}=0.055 \mathrm{~mm}$. The displacement in "2" direction is given as a triangle periodic function (Fig. 5a).

The deformed cube at the time instant $t \in\left(t_{0} ; t_{1}\right)$ can be seen in Fig. $5 \mathrm{f}$. The axial stress response obtained for the HMH plasticity is depicted in Fig. 5b. The axial stress generated for the present constitutive model (Fig. 5c) is in a better agreement with the experimental measurements (e.g. Kowalewski and Szymczak 2009) than the predictions of the classical flow theory.

(a)

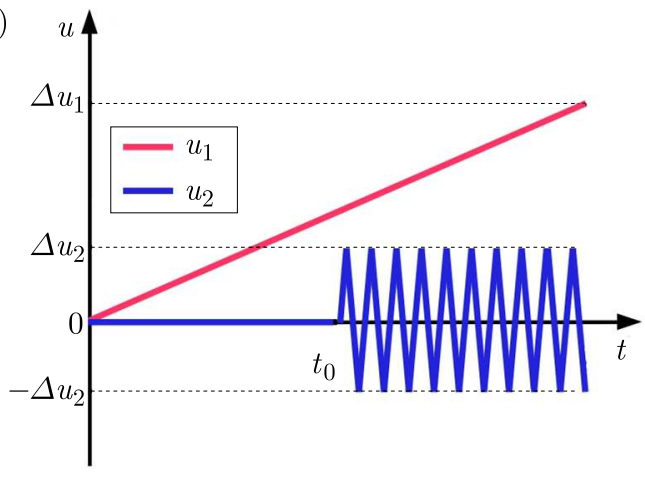

(b)

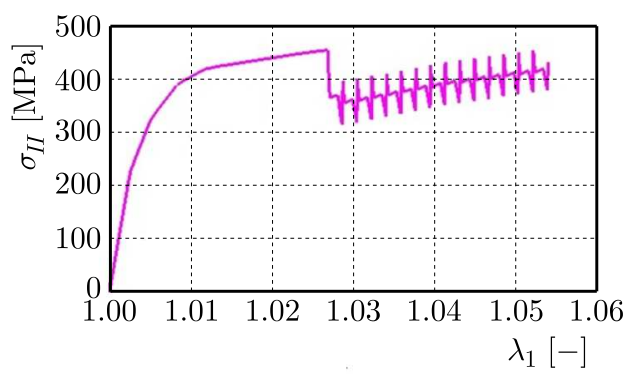

(c)

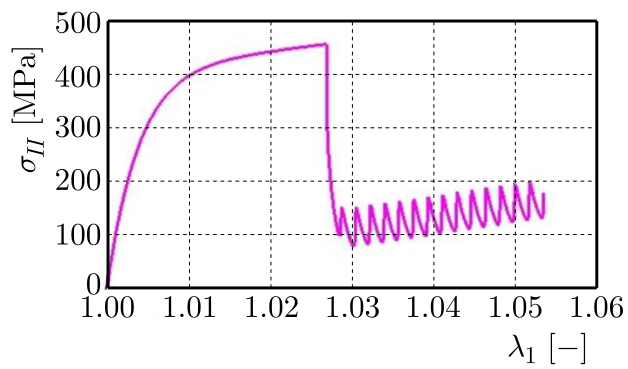

(d)
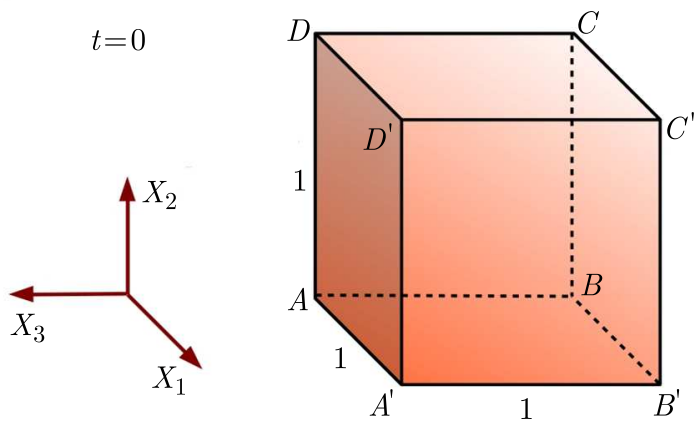

(e)

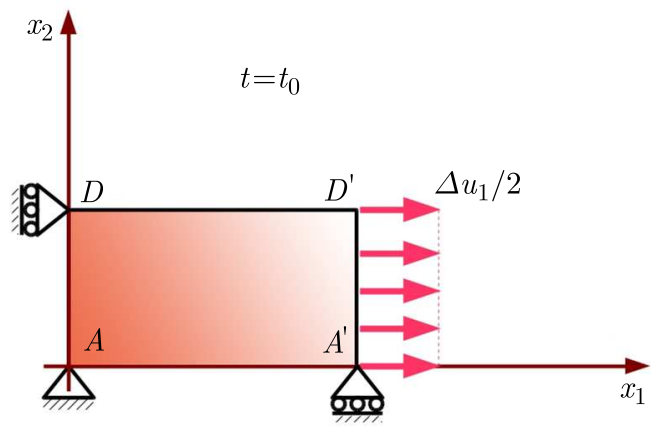

(f)

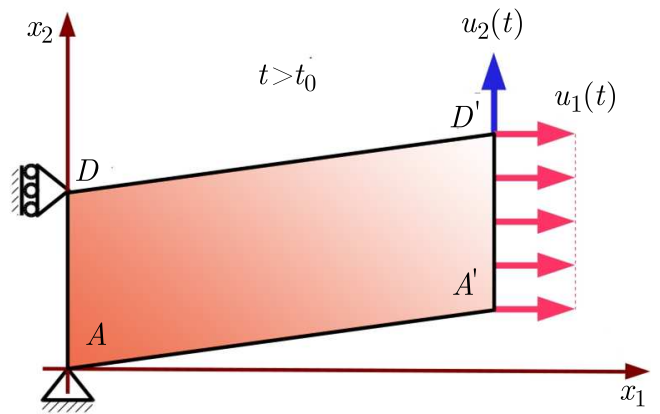

Fig. 5. Combined elongation and shear deformation: (a) displacement functions, (b) shear softening (HMH plasticity), (c) shear softening (UMAT), (d) undeformed cube, (e) deformed cube at the end of stage 1 of the deformation process, (f) deformed cube during stage 2 of the deformation process 


\section{Simulation of nonhomogenous deformation process}

In order to check the performance of the UMAT code in the case of nonhomogenous deformations, a cylinder loaded by the internal pressure has been analyzed. The boundary conditions and cylinder dimensions are depicted in Fig. 6a. Due to the axial symmetry of this problem, only a quarter of the cylinder was taken into account. The FE mesh used C3D8 brick elements and can be seen in Fig. 6b. The loading pressure $p$ was defined to increase linearly until the value of $470 \mathrm{MPa}$ was reached. Subsequently, the unloading process began. A residual stress and strain states remained in the cylinder after the unloading. The material parameter values determined for the Inconel alloy (supersaturated) were utilized (Table 1). The results of FE simulation are gathered in Fig. 7.

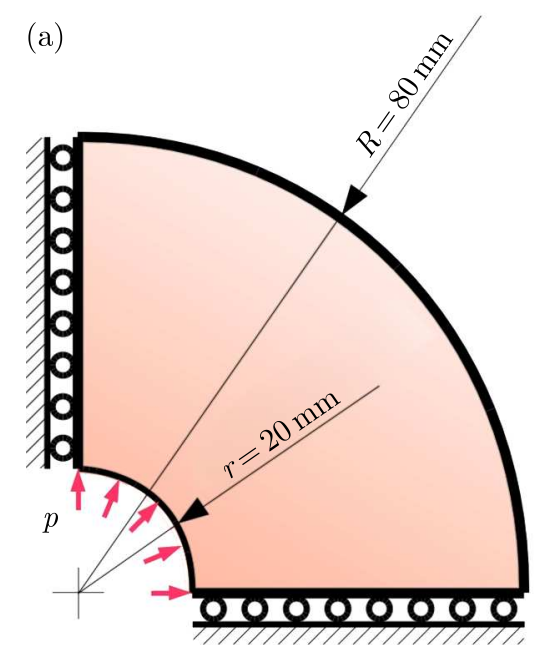

(b)

Fig. 6. Cylinder loaded by internal pressure: (a) boundary conditions, (b) FE mesh
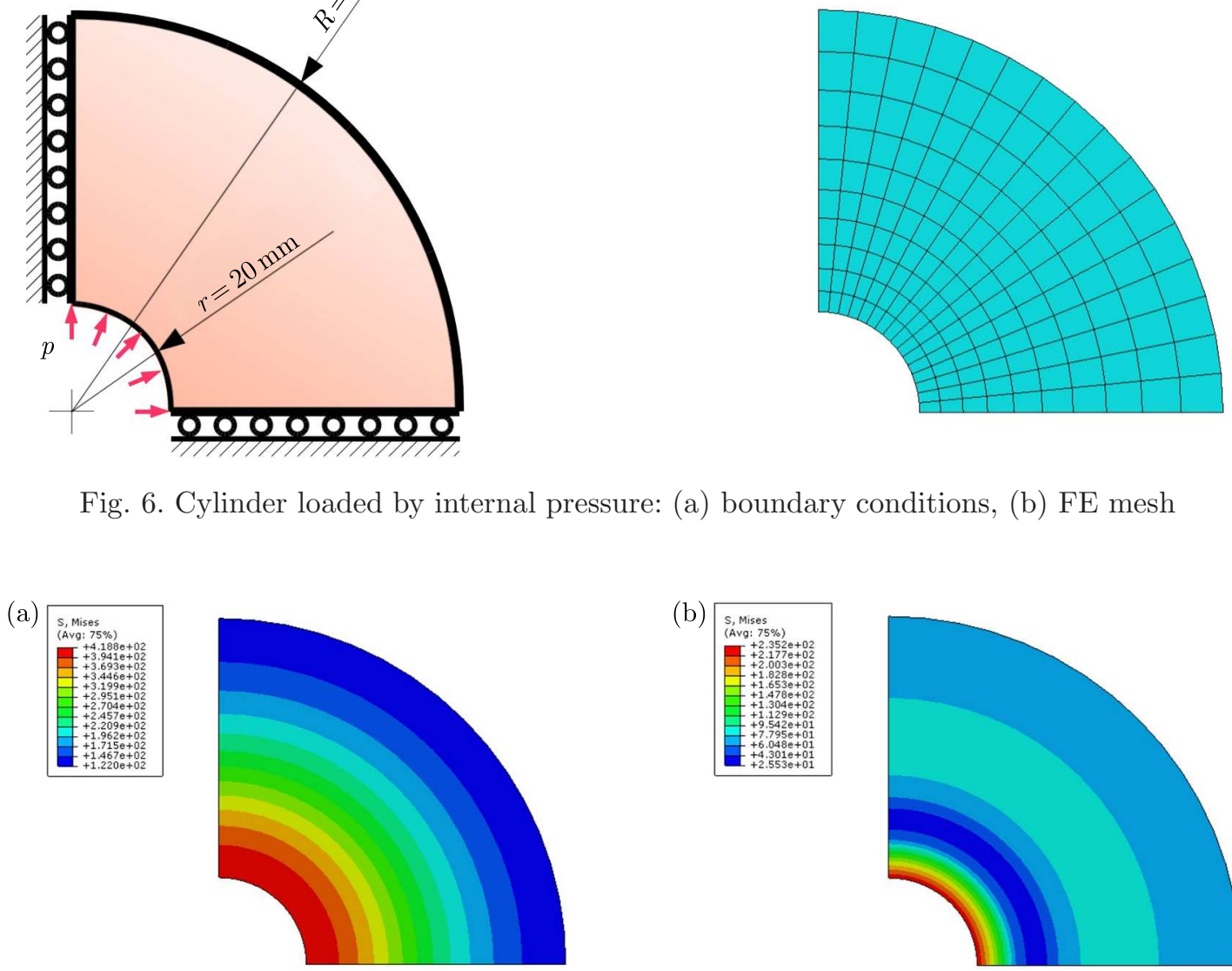

Fig. 7. FE simulation results: (a) HMH stress, (b) HMH residual stress

\section{Conclusions}

In this work, the FE implementation of the elasto-plasticity without the yield surface is discussed. As it is illustrated, in the case of metals, the proposed elasto-plastic model can be implemented into FEM by utilizing the perturbed deformation gradient method. It should be emphasized that the presented framework of modeling is universal in the sense that it provides a uniform constitutive description for both metals and polymers. Furthermore, the number of the material parameters required to be determined is considerably small. The proposed model 
uses 4 constants to describe the mechanical behavior of brass, whereas the HMH flow plasticity needed determination of 16 parameters. The developed constitutive model is capable of describing the Bauschinger effect and shear-softening effects that the classical plasticity fails to capture accurately. What is more, the computation cost turns out to be smaller in the case of using the proposed elastic-plastic model. Further improvement in this matter can be expected after the developed user subroutine is thoroughly optimized.

Acknowledgement

This work was supported by project PBS III/246715/NCBiR.

\section{References}

1. Alkas Yonan S., Soyarslan C., Haupt P., Kwiatkowski L., Tekkaya A.E., 2013, A simple finite strain non-linear visco-plastic model for thermoplastics and its application to the simulation of incremental cold forming of polyvinylchloride (PVC), International Journal of Mechanical Sciences, 66, 192-201

2. Doll S., Schweizerhof K., 2000, On the development of vlumetric strain energy functions, Journal of Applied Mechanics, 67, 17-21

3. Hibbit B., Karlsson B., Sorensen P., 2008, ABAQUS Theory Manual, Hibbit, Karlsson \& Sorensen Inc.

4. Holzapfel G.A., 2010, Nonlinear Solid Mechanics, John Wiley \& Sons Ltd., New York

5. Kästner M., Obst M., Brummund J., Thielsch K., Ulbricht V., 2012, Inelastic material behavior of polymers - Experimental characterization, formulation and implementation of a material model, Mechanics of Materials, 52, 40-57

6. Knowles J. K., 1977, The finite anti-plane shear field near the tip of a crack for a class of incompressible elastic solids, International Journal of Fracture, 13, 5, 611-639

7. Kowalewski Z.L., Szymczak T., 2009, Variation of mechanical parameters of engineering materials under tension due to cyclic deformation by torsion, Engineering Transactions, 57, 113-123

8. KozŁowska B., 2011, Experimental strain and stress analysis in the process of formation and evolution of elastic-plastic zones in construtions (in Polish), Scientific Surveys of Warsaw University of Technology, Mechanics, 239

9. LEE C.F., 1995, Recent finite element applications of the incremental endochronic plasticity, International Journal of Plasticity, 11, 843-865

10. Miene C., 1996, Numerical computation of alghoritmic (consistent) tangent moduli in large-strain computational inelasticity, Computer Methods in Applied Mechanics and Engineering, 134, 223-240

11. Olesiak Z., 1975, On Huber-Mises yield condition (in Polish), Mechanika Teoretyczna i Stosowana, 13, $523-528$

12. Pipkin A.C., Rivlin R.S., 1965, Mechanics of rate-independent materials, ZAMP, 16, 313-327

13. Simo J.C., Hughes T.J.R., 2000, Computational Inelasticity, Springer Verlag Inc., New York

14. Suchocki C., 2015, An internal-state-variable based viscoelastic-plastic model for polymers, Journal of Theoretical and Applied Mechanics, 53, 593-604

15. Suchocki C., Skoczylas P., 2016, Finite strain formulation of elasto-plasticity without yield surface: theory, parameter identification and applications, Journal of Theoretical and Applied Mechanics, 54, 731-742

16. Sun W., Chaikof E.L., Levenston M.E., 2008, Numerical approximation of tangent moduli for finite element implementations of nonlinear hyperelastic material models, Journal of Biomechanical Engineering, 130, 061003-1-061003-7 
17. Sussman T., Bathe K.J., 1987, A finite element formulation for nonlinear incompressible hyperelastic and inelastic analysis, Computers and Structures, 26, 357-409

18. VAlanis K.C., 1971a, A theory of viscoplasticity without a yield surface, Part I: General theory, Archives of Mechanics, 23, 517-534

19. VAlanis K.C., 1971b, A theory of viscoplasticity without a yield surface, Part II: Application to mechanical behavior of metals, Archives of Mechanics, 23, 535-551

Manuscript received October 8, 2016; accepted for print February 15, 2017 